\title{
Comparison between experiments and direct numerical simulations in a channel flow with roughness on one wall
}

\author{
P. BURATTINI I,4, S. LEONARDI ${ }^{2}$, P. ORLANDI \\ AND R. A. ANTONIA ${ }^{4}$ \\ ${ }^{1}$ Physique Statistique et des Plasmas, Université Libre de Bruxelles, B-1050 Brussels, Belgium \\ ${ }^{2}$ Department of Mechanical Engineering, University of Puerto Rico, Mayaguez \\ PR 00681-9045, Puerto Rico \\ ${ }^{3}$ Dipartimento di Meccanica e Aeronautica, Università degli Studi di Roma \\ "La Sapienza," I-00184 Rome, Italy \\ ${ }^{4}$ Discipline of Mechanical Engineering, University of Newcastle, NSW 2308, Australia
}

(Received 10 July 2007 and in revised form 18 January 2008)

The turbulent flow in a two-dimensional channel with roughness on one wall is investigated using experiments and direct numerical simulations (DNS). The elements have a square cross-section with height $k=0.1 H$ ( $H$ is the channel half-width) and a streamwise spacing of $4 k$. The Reynolds number $R e_{\tau_{r}}$, based on the friction velocity at the rough wall and $H$, is in the range 300-1100. Particular attention is given to the rough-wall side. Measured turbulence intensities, length scales, leading terms in the turbulent kinetic energy budget, and velocity spectra are compared with those obtained from the DNS. Close agreement is found, yielding support for the simplifying assumptions in the experiment (notably local isotropy and Taylor's hypothesis) and the adequacy of the spatial resolution in the simulation. Overall, the profiles of the Reynolds normal stresses on the roughness side are almost independent of $R e_{\tau_{r}}$, when normalized by outer variables. Energy spectra at different locations above the rough wall collapse well at high wavenumbers, when normalized by Kolmogorov scales. In contrast to previous studies, a region of negative energy production near the location of the maximum streamwise velocity is not observed. Comparison with a smoothwall channel, at similar values of the friction-velocity Reynolds number, highlights differences only in the streamwise velocity component near the wall.

\section{Introduction}

In practice, any surface in contact with a fluid has a certain roughness, and this directly affects the flow, at least in the proximity of the wall. Often, the roughness has a pattern. Examples include heat exchanger elements, plant canopies, riblets, and dimples on golf balls. In all these cases, the geometry of the solid boundary interferes with the mechanism of heat (e.g. Chang, Liou \& Juan 2005; Wang \& Sunden 2005), scalar or momentum transfer, compared to a smooth wall. The transfers are typically increased when the roughness elements are transverse to the flow.

In the past, research on rough-wall turbulence has focused on several geometrical configurations including: a two-dimensional channel with symmetric (i.e. on both sides) two-dimensional roughness (Krogstad et al. 2005; Bakken et al. 2005; Ashrafian, Andersson \& Manhart 2004; Ashrafian \& Andersson 2006a,b); a two-dimensional 
channel with asymmetric two-dimensional roughness (Cherukat, Na, Hanratty \& McLaughlin 1998; Miyake, Tsujimoto \& Nakaji 2001; Miyake, Tsujimoto \& Nagai 2002; Ikeda 2002; Ikeda \& Durbin 2007; Cui, Patel \& Lin 2003; Leonardi et al. 2003; Nakagawa, Na \& Hanratty 2003; Leonardi et al. 2004; Nagano et al. 2004; Hanjalić \& Launder 1972; Nakagawa \& Hanratty 2003); a two-dimensional channel with asymmetric three-dimensional roughness (Bhaganagar, Kim \& Coleman 2004; Orlandi \& Leonardi 2006); a boundary layer with two-dimensional roughness (Krogstad \& Antonia 1994; Castillo et al. 2004; Djenidi, Elavarasan \& Antonia 1999; Perry, Schofield \& Joubert 1969; Furuya, Miyata \& Fujita 1976; Lee \& Sung 2007); a boundary layer with three-dimensional roughness (Snyder \& Castro 2002; Cheng \& Castro 2002).

In most of the above references for the two-dimensional channel with asymmetric two-dimensional roughness, the ratio of the roughness height to the characteristic length of the outer flow is relatively large, of the order of $10 \%$. Therefore, it is more appropriate to speak of a flow over obstacles (e.g. Jiménez 2004; Finnigan 2000; Raupach, Antonia \& Rajagopalan 1991). In contrast, the classical experiments in a pipe by Nikuradse dealt with sand-grain protrusions at the wall. Moreover, the presence of a second boundary and the streamwise homogeneity may introduce differences between the channel and the boundary layer (e.g. Bakken et al. 2005; Krogstad \& Antonia 1994). Previously, it was suggested that, at a high enough Reynolds number, complete similarity would exist in the outer layer, between rough and smooth walls (see Raupach et al. 1991) and perhaps between the channel flow and the boundary layer.

While the smooth-wall channel has been studied extensively in experiments and DNS, the channel with wall roughness has received less attention. Undoubtedly, compared to a smooth wall, the roughness introduces a different level of complexity because the structures tend to be smaller close to the boundary. In simulations, this requires finer resolutions and, consequently, only recently have accurate computations at moderate Reynolds numbers been possible. Krogstad et al. (2005) and Bakken et al. (2005) reported simulations and experiments in a channel with symmetric, two-dimensional roughness. With the help of quadrant analysis and Reynolds stress anisotropy invariants, these authors showed that the turbulence structures in the outer region may be affected by the geometry of the wall.

Here, we focus on a channel with two-dimensional roughness elements on one wall only. Hanjalic \& Launder (1972, referred to as HL hereafter) were first to consider this geometry experimentally. They tested roughness elements of square cross-section with a spacing-to-height ratio of 10 , and a bulk-flow Reynolds number $\operatorname{Re}_{b}$ in the range $18500-56000$ (in HL, the channel half-width is measured from the base of the roughness). One of the conclusions was that, for different $R e_{b}$, the profiles of each of the three normal Reynolds stresses collapse, when normalized with the friction velocity and a length scale associated with the zero-crossing of the Reynolds shear stress. Detailed measurements of the kinetic energy budget terms and energy spectra were also reported, although the resolution of the probe was marginal and the energy dissipation rate was estimated indirectly, as the closing term in the energy budget equation. DNS for this particular flow and geometry have been made recently by Leonardi et al. (2003). Among other things, they documented the major contribution of the pressure distribution around the elements to the increase in the drag, especially when the roughness pitch is in the range 4 to 12 times the roughness height. This strongly confirmed the earlier observations of Furuya et al. (1976) for a boundary layer roughened with transverse circular rods. 
The interest in the rough-wall channel is partly due to the fact that higher Reynolds numbers can be attained compared to the smooth wall but also because the roughness can significantly increase the momentum and heat transfer rates. Perhaps more generally, the rough-wall geometry may provide valuable insight into the asymptotic (i.e. large Reynolds number) behaviour of the turbulence production in wall-bounded flows.

The present work aims first to compare experiments and direct simulations of a channel flow with roughness on one of the walls, for approximately the same boundary and Reynolds number conditions. In many respects, DNS and experiments complement each other. In simulations, all flow variables are accessible, but adequate spatial resolution and integration time become critical at relatively large Reynolds numbers. In experiments, extremely long velocity signals (of the order of several thousands integral time scales) can be acquired, even at large Reynolds numbers. However, a limited number of variables is accessible, and few can be measured simultaneously, with sufficient space and time resolution. This work promotes an approach based on simultaneous experimental and numerical analyses of the flow. This one-to-one comparison enhances the experimenter's ability to estimate two important quantities, namely the rate at which the mean kinetic energy is dissipated and the friction velocity at the wall. In particular, regarding the dissipation rate, our main conclusion is that, in the equilibrium range, the smallest scales can be effectively considered as isotropic. Using an accurate value of the friction velocity along with an appropriate length scale certainly determines whether or not turbulence distributions can be rescaled into a general form. The present analysis highlights what these scaling quantities are for a channel flow with roughness on one wall.

A second point that we consider in detail is the kinetic energy budget. In HL, it was conjectured that the asymmetry of the boundary conditions may induce a region of negative turbulent energy production. In fact, if the locations where the mean velocity is maximum and the shear stress is zero are not coincident, the possibility that the mean field can extract energy from the turbulent field may exist, albeit in a limited part of the flow. Although such an occurrence does not contradict any physical law, it is somewhat counterintuitive since, for instance, it invalidates completely the eddy viscosity concept (e.g. Mathieu \& Scott 2000). Other flows show limited regions of negative energy production, including the near field of a turbulent round jet (Zaman \& Hussain 1980), the plane wall jet (Mathieu \& Scott 2000), the flow in an annulus (Chung, Rhee \& Sung 2002), the rotating channel and pipe (Jakirlic, Hanjalic \& Tropea 2002), the Rayleigh-Bénard convection cell (Liberzon et al. 2005). For the asymmetric channel, support for a negative energy production region remains lacking. Although HL and Thurlow \& Klewicki (2000) showed this possibility in experiments, recent DNS by Nagano et al. (2004) indicated that the zero-crossings of the shear stress and location of maximum streamwise velocity differed by only about $0.8 \%$ to $2.8 \%$. Nagano et al. (2004) hypothesized that this could depend on their low value of $R e_{b}$. Also Leonardi, Orlandi \& Antonia (2005) found that the two loci were almost coincident. If the negative energy production region were significant, one would expect to see some difference between the boundary layer and the asymmetric channel.

A further aspect, related to the asymmetry of the boundary conditions in the present flow, is the approach of the velocity profiles towards an asymptotic state. Since the friction coefficient on the rough wall is arguably independent of the Reynolds numberbecause it is largely due to the pressure drag - the evolution of the turbulent profiles with $R e_{b}$ may depend solely on the behaviour of the flow near the smooth wall. The friction coefficient on that side varies with the Reynolds number. 


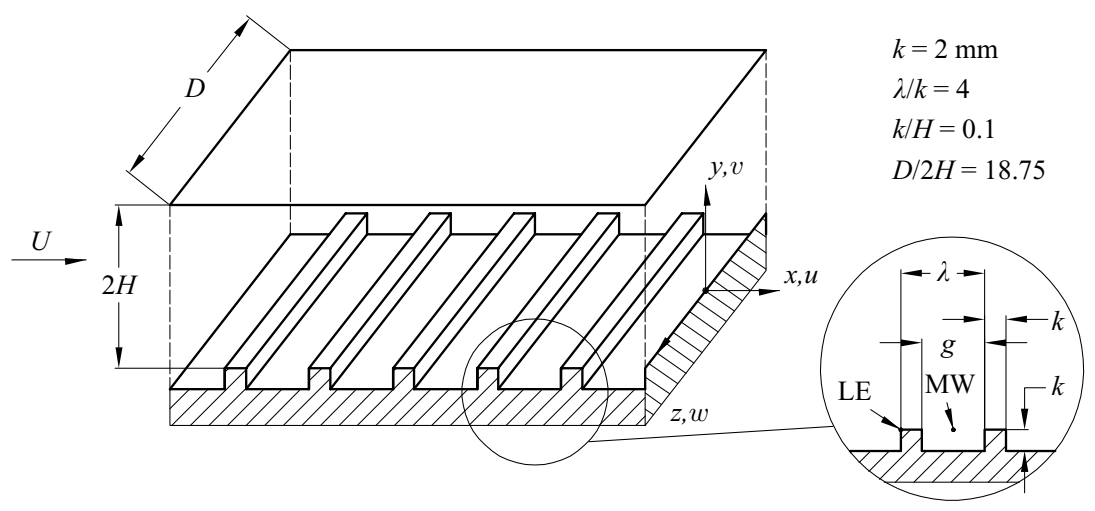

FIGURE 1. Schematic of the two-dimensional channel with the roughness geometry. The origin of the Cartesian coordinate system is at mid-span, the $x$-axis along the streamwise direction, and the $z$-axis on the leading edge of an element. The blow-up shows the two streamwise locations (leading edge LE and midway MW) where the measurements are made. $k$, roughness height; $\lambda$, roughness pitch; $g$, roughness gap; $H$, channel half-width; $D$, channel span; $U$, streamwise velocity.

Lastly, we consider the turbulent energy spectra. In the literature regarding channel flow with roughness, these have not received the same attention as other basic turbulent statistics. As previously noted, the data of HL suffered from limited spatial resolution of the hot wire and consequently new measurements are desirable. More recently, Nakagawa \& Hanratty (2003) and Nakagawa et al. (2003) showed that the velocity spectra, measured over a wavy wall in an asymmetric channel, collapse on outer variables, when the distance from the wall exceeds about $0.2 y_{U}\left(y_{U}\right.$ is the location of the streamwise velocity maximum). Here, this suggestion is tested and compared with the classical Kolmogorov scaling, at various distances from the wall and Reynolds numbers.

The paper presents detailed experimental distributions, corroborated by DNS results, of statistical moments (up to the fourth order) of velocity fluctuations, length scales and power spectra, and their variation with the Reynolds number. The ensemble of these quantities has never been presented before in detail and with high accuracy for the present geometry. And yet they would be invaluable in the context of flow modelling with simplified approaches, such as one-point closures or large-eddy simulations.

\section{Experimental details}

The experiment is performed in an open-circuit, blower-type wind tunnel. The blower and diffuser are connected through a flexible section, which isolates the vibrations of the fan. Honeycomb and grids are fitted in the plenum chamber to reduce the turbulence intensity and straighten the flow. A two-dimensional contraction (area ratio 14:1) precedes the working section, which is rectangular and $7.32 \mathrm{~m}$ long. Roughness elements are attached, transversely to the flow (figure 1), on only one side of the channel, over the last $3.16 \mathrm{~m}$, or $43 \%$ of the working section. The elements, made of brass, have a square cross-section (dimension $k=2 \mathrm{~mm}$ ) and are equally spaced in the streamwise direction by a pitch $\lambda=4 k$. Such separation has been chosen because it was previously studied by some of us (Leonardi et al. 2003) and because it displays several features in common with larger pitch values. The channel half-width, 


$\begin{array}{lcccccc}\text { Case } & R e_{b}=\frac{U_{b} H}{v} & \ell_{w}^{+}=\frac{\ell_{w} u_{\tau_{r}}}{v} & f_{s}[\mathrm{kHz}] & \min \left(\frac{T_{s}}{T_{u}}\right) & \min \left(\frac{f_{f}}{f_{K}}\right) & \max \left(\frac{\ell_{w}}{\eta}\right)^{\dagger} \\ \text { E1 } & 3600 & 3.8 & 3.2 & 840 & 0.7 & 2.2 \\ \text { E2 } & 7100 & 7.9 & 6.3 & 833 & 0.42 & 3.9 \\ \text { E3 } & 13000 & 14 & 12.6 & 1400 & 0.27 & 5.7\end{array}$

TABLE 1. Main experimental conditions $\ell_{w}$ is the length of the single wire. Resolution parameters (virtually all columns) refer to the single wire with diameter $d_{w}=1.27 \mu \mathrm{m}$. ${ }^{\dagger}$ The maximum is located near the rough wall.

measured from the roughness crest, is $H=20 \mathrm{~mm}$, while the span of the section $D$ is $750 \mathrm{~mm}$. The aspect ratio $D / 2 H=18.75$ is large enough to ensure two-dimensionality of the flow. Three bulk-flow Reynolds numbers $R e_{b}=H U_{b} / v\left(U_{b}\right.$ is the bulk velocity and $v$ is the kinematic viscosity of air) are tested. The three experimental cases are denoted E1, E2 and E3, see table 1. Nearly the same values of $R e_{b}$ are used in the simulations.

The origin of the Cartesian coordinate system is at mid-span, with the $x$-axis parallel to the streamwise direction, and the $y$-axis on the leading edge of a roughness element, figure 1. (The flow is homogeneous in the streamwise direction, when averaged over the roughness pitch, so that the origin of the coordinate system, with respect to the contraction, need not be specified.) The 'true' origin for $y$ is often taken to lie somewhere between the base and the apex of the roughness elements, either on the basis of momentum balance considerations (e.g. Leonardi et al. 2003) or by assuming the validity of a mean velocity log-law. Here we have simply taken the origin at the plane of the roughness elements; this choice should not be critical since the focus is primarily on comparing experimental and numerical results. The fluctuating velocities are denoted by $u, v, w$; corresponding capital letters refer to averaged values, while the overbar represents time-averaging. In the experiments, averages are performed in time; in simulations, they are performed in time and over the $x$ - and $z$-axes.

Hot-wire anemometers, with single and $\mathrm{X}$-wires, are used for measuring the streamwise, wall-normal, and spanwise velocity components. Two streamwise locations are probed, one corresponding to the leading edge (LE) of the 10th element from the exit, and a second midway (MW) between the 10th and the 11th element (see figure 1). These locations are easily accessible from the exit of the tunnel, while being sufficiently inside the working section to avoid end-effects. The single wire is used to acquire a second, independent estimate of the streamwise velocity, since, compared to the X-wire, it has a better spatial resolution (especially close to the wall where the mean velocity gradient is higher) and its calibration is more reliable. Differences, between single and X-wire estimates of large-scale quantities, are within a few percent. For the X-probe, the lateral separation of the wires is $0.76 \mathrm{~mm}$ and their diameter is $d_{w}=2.54 \mu \mathrm{m}$; for the single wire, $d_{w}=1.27 \mu \mathrm{m}$. In both cases, the length of the wire $\ell_{w}$ is $\simeq 200 d_{w}$, and the overheat ratio 1.5. A look-up table calibration (Burattini \& Antonia 2005), covering a range of velocities and angles of $0-14 \mathrm{~m} \mathrm{~s}^{-1}$ and $\pm 40^{\circ}$ respectively, is used for the X-probe, whereas the single wire is calibrated in the range $0-14 \mathrm{~ms}^{-1}$, fitting its response to a third-order polynomial. Calibrations are re-checked at the end of an experiment. A Pitot tube connected to a pressure transducer (Setra model 239, full scale 0.5 in. $\mathrm{H}_{2} \mathrm{O}$ or $\simeq 125 \mathrm{~Pa}$, accuracy $\pm 0.14 \%$ of full scale) provides the reference velocity. The signal from the anemometers is first 


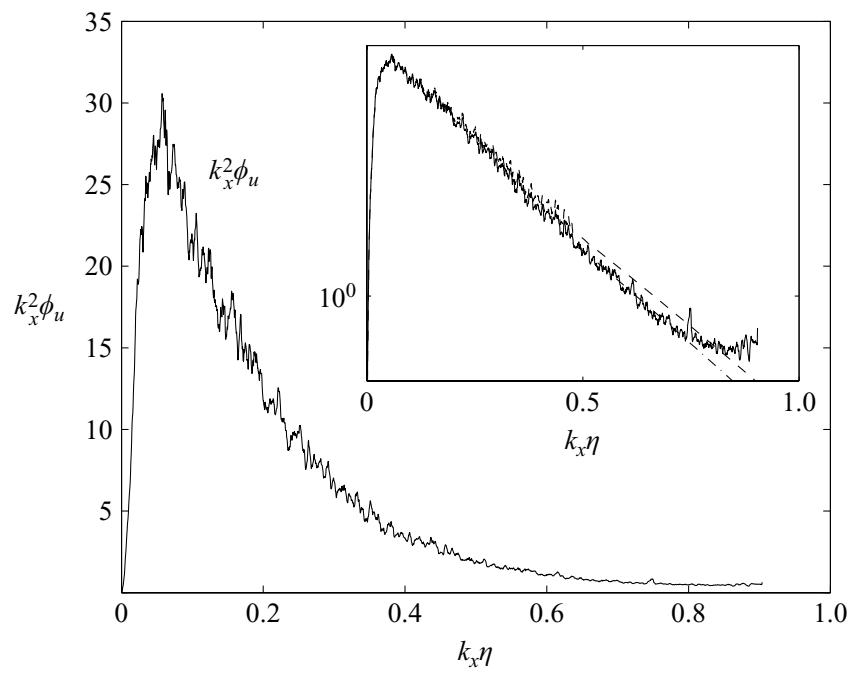

FIGURE 2. Spectrum of $\partial u / \partial x$, measured with a single wire for E1, $y / H=0.05\left(\phi_{u}\right.$ is the power spectrum of the velocity component $u$, see (4.7)). Inset: semilogarithmic plot of the spectrum. -, original distribution; -., distribution filtered from noise at large wavenumbers; -- , distribution filtered from noise and corrected for spatial resolution of the probe.

conditioned through buck-and-gain modules, then low-pass filtered at a frequency $f_{f}$ (corresponding to the rise of electronic noise and close to the Kolmogorov frequency $f_{K}=U / 2 \pi \eta, \eta=v^{3 / 4} \epsilon^{-1 / 4}$ being the Kolmogorov length scale, and $\epsilon$ the mean energy dissipation rate), and finally sampled at $f_{s}=2 f_{f}$; the resolution of the digitization is 16 bit. The sampling time $T_{s}$, expressed in terms of the integral time scale $T_{u}$ (see later for its definition), is also included in table 1.

A correction for the attenuation, due to finite resolution of the single hot wire, is made using a variation of Wyngaard's (1968) approach, see below. The worst-case conditions, in terms of resolution, are listed in table 1. Details of the measurement of the wall shear stress on the rough wall $u_{\tau_{r}}$ are given in $\S 2.2$.

\subsection{Mean energy dissipation rate}

The experimental evaluation of the turbulent kinetic energy dissipation rate is typically affected by several uncertainties. These arise from electronic noise contamination, sampling discretization error, finite spatial resolution of the probe, use of Taylor's hypothesis, and incomplete knowledge of the energy dissipation rate tensor. Whilst the first three issues have been alleviated here, the latter two pose almost insurmountable difficulties in experiments. In this respect, the DNS has provided a posteriori validation of the simplifying assumptions made in the measurements.

The isotropic mean energy dissipation rate is given by

$$
\epsilon_{i s o}=15 v \overline{(\partial u / \partial x)^{2}}
$$

(in the experiments, Taylor's hypothesis $\partial / \partial x=-U^{-1} \partial / \partial t$ is assumed). The spectrum of $\partial u / \partial x$ is given in figure 2, for a location close to the rough wall, where the resolution is marginal. Although the distribution is free from noise spikes and closes almost adequately at high wavenumbers, denoted by $k_{x}(=2 \pi f / U, f$ is the frequency), electronic noise and discretization error result in a spurious upturn of the distribution at large $k_{x}$, near $k_{x} \eta=0.7$ in the inset of the figure. Such contamination, which increases the estimated $\epsilon_{i s o}$, can be filtered out by extending heuristically the energy 
spectrum in the far-dissipative range. This range is modelled with the function $\exp \left(a\left(k_{x} \eta\right)^{2}+b\right)$, in a similar manner to that documented by Martinez et al. (1997) for the three-dimensional energy spectrum. The extrapolation (see inset) effectively removes the high-wavenumber noise.

The energy and dissipation spectra are then compensated for the limited resolution of the probe according to method of Wyngaard (1968) but using the spectrum of Martinez et al. (1997). Figure 2 shows the final corrected energy spectrum. The resulting corrections to the measured values of $\epsilon_{i s o}$ are in the ranges 3-6\% for E1, 4-10\% for E2, and $14-24 \%$ for E3 (the larger values are for locations closer to the rough wall). The $\mathrm{X}$-wire data are not corrected for spatial resolution since they are used mainly to estimate large-scale quantities.

\subsection{Wall shear stress}

In experiments, the direct estimate of the wall shear stress from the slope of the mean velocity profile,

$$
\tau_{w}=\rho u_{\tau}^{2}=\left.\rho v \frac{\partial U}{\partial y}\right|_{y=0}
$$

( $\rho$ is the fluid density), presents some difficulties. This is true for a smooth wall, and even more so for a rough wall, where the mean velocity $U$ varies also with $x$, very near the roughness. A more convenient way of estimating $\tau_{w}$ is via the $x$-momentum balance equation,

$$
\frac{1}{\rho} \frac{\mathrm{d} P}{\mathrm{~d} x}=v \frac{\mathrm{d}^{2} U}{\mathrm{~d} y^{2}}-\frac{\mathrm{d}}{\mathrm{d} y} \overline{u v} .
$$

Here, the flow variables are averaged not only in time but also over one roughness wavelength but, for simplicity, we keep the old variable notation. Equation (2.3) can be rewritten

where

$$
\frac{\mathrm{d} P}{\mathrm{~d} x}=\frac{\mathrm{d} \tau}{\mathrm{d} y}
$$

$$
\tau=\rho \nu \frac{\mathrm{d} U}{\mathrm{~d} y}-\rho \overline{u v}
$$

is the total shear stress. Since, in the channel, $(\mathrm{d} P / \mathrm{d} x)$ is constant, from (2.4) it follows that $\tau$ is linear across the section, i.e.

$$
\tau(y)=\tau_{r}+\left(\tau_{s}-\tau_{r}\right) \frac{y}{2 H}
$$

( $r$ indicates the rough side, $s$ the smooth side, see figure 3). Away from the walls, the main contribution to $\tau$ comes from $\rho \overline{u v}$, which can be measured reliably with an $\mathrm{X}$-wire. Therefore, the distribution of the Reynolds shear stress can be extrapolated linearly to the walls to yield $\tau_{s}$ and $\tau_{r}$.

Further, integration of (2.4) yields $\left(\tau_{r}-\tau_{s}\right)=-2 H(\mathrm{~d} P / \mathrm{d} x)$ or, considering the individual contributions of the wall friction to the momentum balance,

$$
\begin{aligned}
\tau_{r} & =-y_{\tau} \frac{\mathrm{d} P}{\mathrm{~d} x} \\
\tau_{s} & =\left(2 H-y_{\tau}\right) \frac{\mathrm{d} P}{\mathrm{~d} x} .
\end{aligned}
$$

Here, $y_{\tau}$ is the location of zero-crossing of $\tau(y)$, which practically coincides with $y_{u v}$, the location of the zero-crossing of $\rho \overline{u v}$ (figure 3). Equations (2.7)-(2.8) - which 


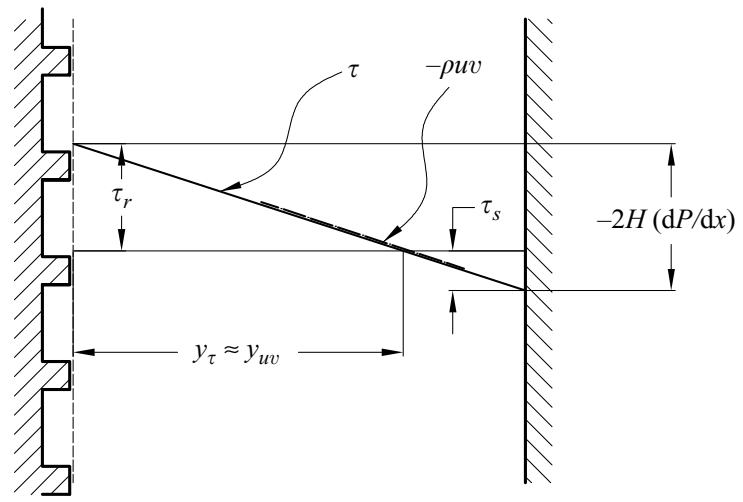

FIGURE 3. Schematic profile of the Reynolds shear stress in a channel with asymmetric roughness and the method for determining the wall shear stresses.

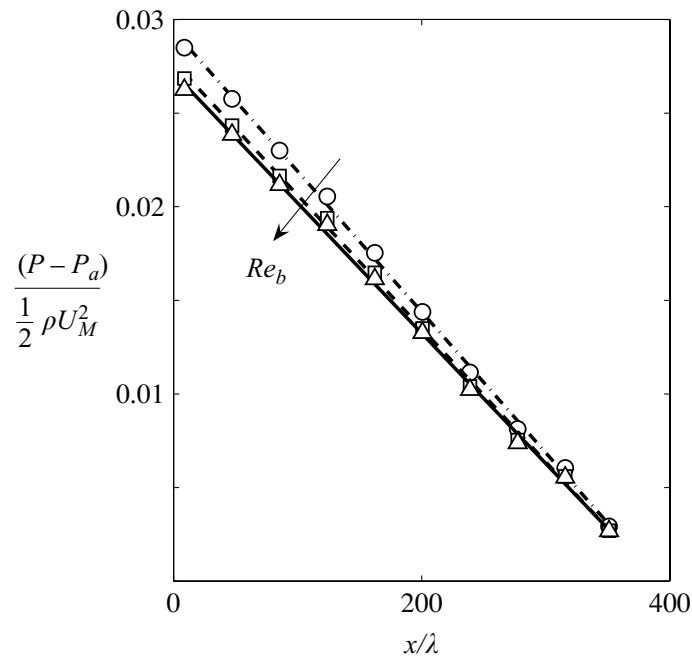

FIGURE 4. Streamwise variation of static pressure measured with pressure taps on the smooth wall. $P_{a}$ is the ambient pressure and $U_{M}$ is the streamwise velocity maximum. $\cdot-\cdot-\cdot \bigcirc$, E1; $---\square, \mathrm{E} 2 ;-\triangle, \mathrm{E} 3$. Lines are linear fits to the data used to estimate $\mathrm{d} P / \mathrm{d} x$.

express the equilibrium of the fluid regions between the plane of zero shear stress and the rough or smooth walls (Vlachogiannis \& Hanratty 2004; Leonardi et al. 2005) provide an independent check on the estimates of $\tau_{r}$ and $\tau_{s}$. In the experiment, the pressure drop (measured from pressure taps along the smooth wall, figure 4) is linear, to a close approximation. Incidentally, this suggests that the roughness fetch is enough to achieve a fully developed flow. A slight dependence on the Reynolds number remains. In the following, the frictional velocity at the rough wall is estimated from $u_{\tau_{r}}^{2}=\tau_{r} / \rho$. This velocity is subsequently used in wall-based normalizations and $R e_{\tau_{r}}$.

As noted previously, the present analysis neglects the role of the dispersive stress (e.g. Finnigan 2000; Ashrafian et al. 2004) which arises from the streamwise waviness of $U$ and $V$ induced by the periodic roughness geometry. The effect of this stress is usually limited to a region near the wall, perhaps within one roughness height (Ikeda 2002), although it may increase with $\lambda / k$ (Ashrafian et al. 2004). 


$\begin{array}{cccccc} & R e_{b}=\frac{H U_{b}}{v} & \text { Resolution } & \text { Fields } & \Delta y^{+}=\frac{\Delta y^{\dagger} u_{\tau_{r}}}{v} & \max \left(\frac{\Delta y}{\eta}\right)^{\ddagger} \\ \text { D1 } & 2800 & 451 \times 151 \times 193 & 80 & 0.8 & 2.3 \\ \text { D2 } & 6900 & 601 \times 201 \times 257 & 80 & 1.6 & 2.8 \\ \text { D3 } & 12000 & 801 \times 321 \times 385 & 80 & 1.8 & 2.6\end{array}$

TABLE 2. Main conditions in the simulations. The size of the computational box is $8 H \times 2 H \times \pi H$, in the $x$-, $y$ and $z$-directions. ${ }^{\dagger}$ Evaluated on the plane of the crests; $¥$ the maximum is at $y \simeq 0.7 H$.

\section{Numerical procedure}

The non-dimensional Navier-Stokes equations coupled with the incompressibility condition,

$$
\begin{aligned}
\frac{\partial\left(U_{i}+u_{i}\right)}{\partial t}+\left(U_{j}+u_{j}\right) \frac{\partial\left(U_{i}+u_{i}\right)}{\partial x_{j}} & =-\frac{1}{\rho} \frac{\partial(P+p)}{\partial x_{i}}+\frac{\partial^{2}\left(U_{i}+u_{i}\right)}{\partial x_{j}^{2}} \\
\frac{\partial\left(U_{i}+u_{i}\right)}{\partial x_{i}} & =0,
\end{aligned}
$$

are discretized in an orthogonal coordinate system (as in $\S 2$, upper- and lower-case variables represent mean and fluctuating quantities, although in the present section the index notation is used) with a staggered central second-order finite-difference approximation. In (3.1), a constant pressure gradient $\mathrm{d} P / \mathrm{d} x$ is prescribed, so as to maintain a constant flow rate. Here, only the main features of the numerical method are recalled (see Orlandi 1999, for details). The discretized system is advanced in time using a fractional-step method with viscous terms treated implicitly and convective terms explicitly. The large sparse matrix resulting from the implicit terms is inverted by an approximate factorization technique. At each time step, the momentum equations are advanced with the pressure at the previous step, yielding an intermediate non-solenoidal velocity field; then, by solving Poisson's equation, the field is made solenoidal. A hybrid, low-storage, third-order Runge-Kutta scheme is used to advance the solution in time.

The roughness is treated by the immersed boundary (IB) technique described in Leonardi et al. (2006b), and periodic boundary conditions are applied in the $x$ - and $z$-directions; zero velocity is imposed at the walls. The applicability and validity of the IB method have been extensively discussed in the literature. It allows an accurate and efficient treatment of the solid boundaries that cannot yet be matched by other approaches, such as the body-fitted method. Strong support for the IB method in a geometry similar to that considered here was provided in Leonardi et al. (2003). They showed that the pressure distribution around circular elements placed on the wall agreed closely with that measured in the experiment of Furuya et al. (1976). The number of velocity fields used for time averaging is 80 (the velocity fields are two time units apart, the unit being $H / U_{1}$ ). Statistics performed using 40 fields showed negligible differences. The initial velocity field (at the start of the computation) was taken from a smooth-wall turbulent channel flow database at $R e_{\tau}=380$. In order to achieve the desired flow rate, about 300 time units were discarded before averaging was applied. A grid-independence test was performed for the case at the intermediate Reynolds number, see Leonardi et al. (2006b). Table 2 lists the main conditions in the simulations, including the number of grid points in the $x$-, $y$-and $z$-directions, 


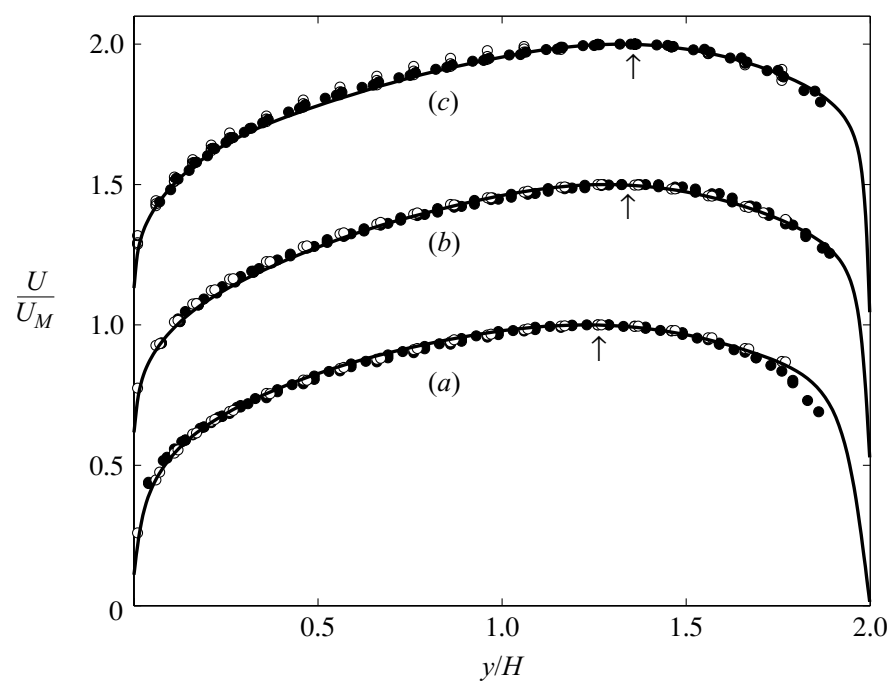

FIGURE 5. Mean streamwise velocity. Symbols: experiments; lines simulations. Filled symbols: X-wire; empty symbols: single wire. (a) E1 and D1; (b) E2 and D2; (c) E3 and D3. Profiles $(b)$ and $(c)$ are shifted upwards by 0.5 and 1 . Arrows indicate $y_{U} / H$ in experiments (values for the simulations are listed on table 3 ).

the number of independent fields for the statistical analyses, and the worst-case resolution in the wall-normal direction (normalized by inner and Kolmogorov scales). Note that, while in the experiments the resolution is worst close to the wall, in the simulations the clustering of grid points near the roughness shifts the point with the poorest resolution of the small scales to the location $y / H \simeq 0.7$. Furthermore, in the simulations, $\epsilon$ is estimated from the complete dissipation tensor, i.e.

$$
\epsilon=\frac{v}{2} \overline{\left(\frac{\partial u_{i}}{\partial x_{j}}+\frac{\partial u_{j}}{\partial x_{i}}\right)^{2}}
$$

instead of (2.1).

\section{Results}

\subsection{Velocity statistics}

The distributions of the mean streamwise velocity (figure 5), as obtained from the experimental and numerical data, are in good agreement with each other. Note that the values from the single and X-wire overlap. While the profiles are skewed towards the smooth wall (the rough wall is more effective at slowing down the flow), there is no discernible difference between profiles at LE and MW, implying a negligible modulation of the mean flow due to the roughness. The numerical results of Cui et al. (2003) and Nagano et al. (2004) indicated that, for the flow in an asymmetric channel of similar geometry to that considered here, the streamwise variation of the mean velocity is rather small (see figure 9 of each paper), although it may become more important at larger $\lambda / k$. The location $y_{U}$ where $U$ is maximum (indicated by an arrow in figure 5) is $\simeq 1.32 \mathrm{H}$, for the two experimental cases at the higher Reynolds numbers, suggesting that $y_{U}$ may have reached its asymptotic value (see table 3 ). It is perhaps not a coincidence that, for E2, the roughness Reynolds number $k^{+}=k u_{\tau_{r}} / v$ is just below 70 - the limit for fully rough flows over sand-grain roughness. 


$\begin{array}{lccccccc}\text { Case } & R e_{b}=\frac{U_{b} H}{v} & R e_{\tau_{r}}=\frac{u_{\tau_{r}} H}{v} & R e_{\tau_{s}}=\frac{u_{\tau_{s}} H}{v} & k^{+}=\frac{k u_{\tau_{r}}}{v} & y_{U} / H & u_{\tau_{r}} / U_{M} & u_{\tau_{r}} / u_{\tau_{s}} \\ \text { E1 } & 3600 & 300 & 230 & 30 & 1.24 & 0.073 & 1.31 \\ \text { E2 } & 7100 & 620 & 430 & 62 & 1.32 & 0.074 & 1.44 \\ \text { E3 } & 13000 & 1120 & 760 & 112 & 1.33 & 0.072 & 1.48 \\ \text { D1 } & 2800 & 260 & 190 & 26 & 1.22 & 0.078 & 1.38 \\ \text { D2 } & 6900 & 634 & 430 & 63 & 1.27 & 0.076 & 1.46 \\ \text { D3 } & 12000 & 1094 & 730 & 109 & 1.27 & 0.076 & 1.50\end{array}$

TABLE 3. Main results for measurements (E1, E2, E3) and simulations (D1, D2, D3).

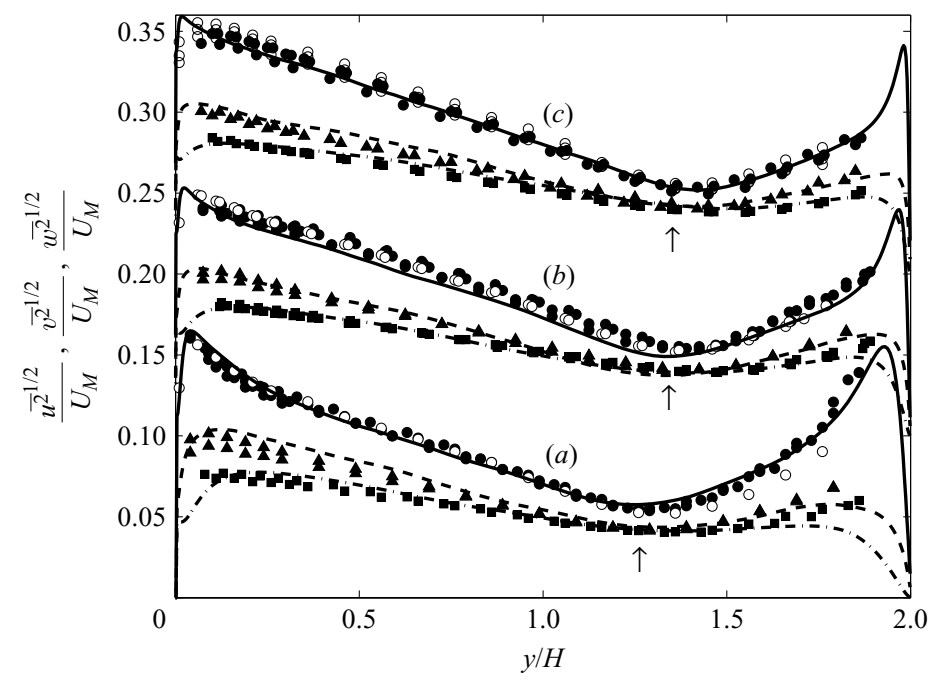

FIGURE 6. RMS turbulence intensities. (a) E1 and D1; (b) E2 and D2; (c) E3 and D3. (b) and $(c)$ are shifted upwards by 0.1 and 0.2. Symbols: experiments; lines simulations. Filled symbols: X-wire; empty symbols: single wire. $\boldsymbol{\bullet}, 0,-, u ; \mathbf{\square},-, v ; \mathbf{\Lambda},--, w$. Arrows indicate $y_{U} / H$ from experiments. Repeated symbols are for different streamwise positions (MW and LE).

There is good agreement also between measured and calculated turbulence intensities, figure 6. The maximum intensities occur near the rough wall, while the locations of the minima are near, but not coincident with, $y_{U}$, particularly for $v$ and $w$ (noted also in HL). Clearly, this contrasts with the outer layer of the symmetric channel (smooth or rough), where the two locations coincide. The relative magnitudes of the three components are such that $\overline{u^{2}}>\overline{w^{2}}>\overline{v^{2}}$ everywhere, as in a smooth channel. The profiles of $\overline{u^{2}}$ near the rough wall depend markedly on $R e_{\tau_{r}}$. For the smallest $R e_{\tau_{r}}$, the distribution of ${\overline{u^{2}}}^{1 / 2}$ has a wide region $(0.05 \lesssim y / H \lesssim 0.5)$ where the curvature is positive, as in the vicinity of a smooth wall. As $R e_{\tau_{r}}$ increases, this region shrinks, and the profile of ${\overline{u^{2}}}^{1 / 2}$ becomes almost linear for $0.1 \lesssim y / H \lesssim y_{u v} / H$, similarly to $v$ and $w$. This suggests differences in the turbulence generation at the rough wall, since the second derivative of the turbulence intensities is linked to the viscous diffusion. (In the experiments, the limited resolution of the X-probe can attenuate the gradient of the actual profile, very near the wall.) Like $U$, no significant dependence on the streamwise position (i.e. LE or MW) is observed, for the normal 


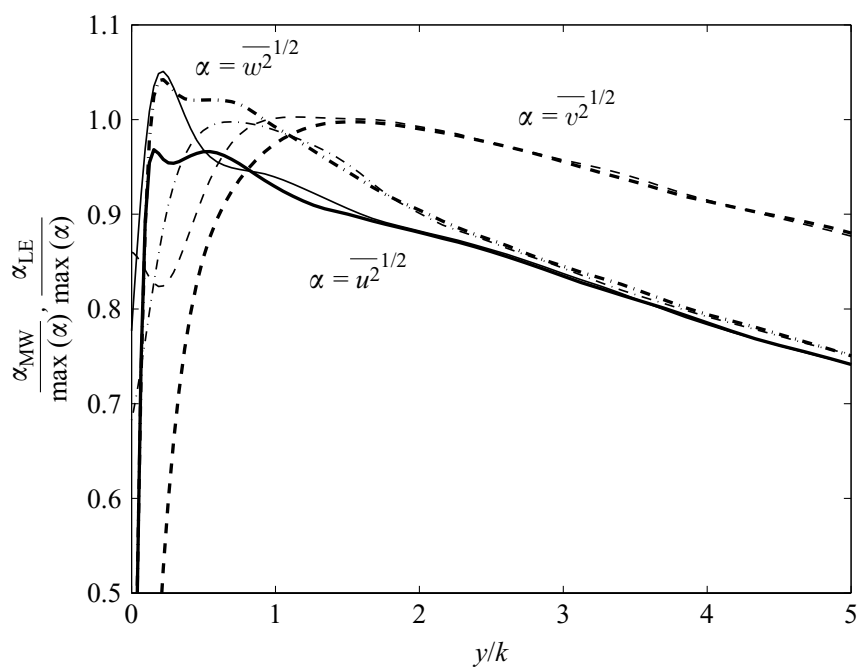

FIGURE 7. Ratio of the phase-averaged quantities (at MW, thin lines, and LE, thick lines) to the maximum of those ensemble averaged along the streamwise direction.,$- u ;--, v ; \cdot-, w$.

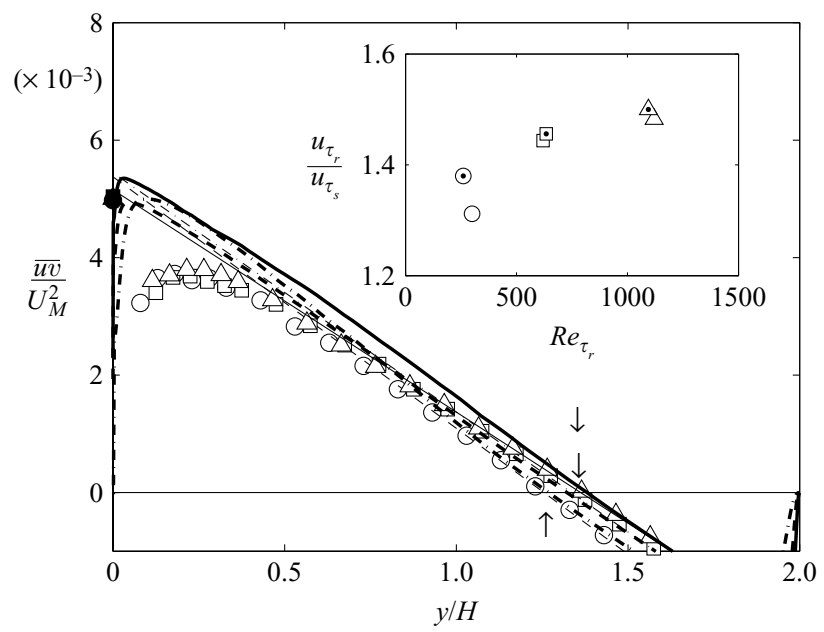

FIgURE 8. Reynolds shear stress. $\bullet, \bigcirc: \mathrm{E} 1 ; \mathbf{\square}, \square$ : E2; $\boldsymbol{\Delta}, \triangle$ : E3. Empty symbols: from X-wire; filled symbols (at $y=0$ ): estimate of $u_{\tau_{r}}^{2}=\tau_{r} / \rho$ from pressure gradient. Thin lines are a linear fit to the experimental data for $y / H \underset{\tau_{r}}{\gtrsim} 0.5 ; \cdot-, \mathrm{E} 1 ;--, \mathrm{E} 2 ;-, \mathrm{E} 3$. Arrows indicate $y_{U} / H$ in experiments. Thick lines are $\frac{y}{u v} / U_{M}^{2}$ in simulations: $\cdot-, \mathrm{D} 1 ;--, \mathrm{D} 2 ;-, \mathrm{D} 3$. Inset: ratio of friction velocities at the wall, for the three cases; dotted symbols: DNS; empty symbols: experiments.

stresses. This is further confirmed by the numerical profiles of the turbulent intensities averaged in phase in the streamwise direction, figure 7 . Their difference with respect to the profile that is ensemble averaged over the length of the channel becomes negligible for $y / k>1.5$.

The Reynolds shear stress $\overline{u v}$ (figure 8 ) varies linearly, away from the walls. The contributions to $\tau$ from the viscous and dispersive stresses must therefore decay rapidly, so that $\rho \overline{u v}$ accounts for most of the total shear stress. Consequently, a linear extrapolation to $y=0$ (the plane of the crests) of the straight portion of $\overline{u v}$ readily 
provides the friction velocity at the rough wall. (In the vicinity of the rough wall, the resolution of the X-wire is critical because of the steep velocity gradients, and the difference with the numerical data is largest.) The resulting value of $\tau_{r}$ is close to that inferred from the pressure drop, see figure 8. For the three experimental cases, the ratio $u_{\tau_{r}} / U_{M}$ remains nearly constant and $\simeq 0.073$, implying that the friction coefficient of the rough wall (proportional to $u_{\tau_{r}}^{2} / U_{M}^{2}$ ) is independent of $R e_{\tau_{r}}$, in the range considered. This is in accord with earlier observations from simulations (Miyake et al. 2002; Cui et al. 2003; Leonardi et al. 2003) and experiments (Bakken et al. 2005). In each case, the major contributor to drag is pressure, and therefore the friction coefficient is expected to be largely independent of the velocity. On the other hand, the friction coefficient at the smooth wall varies with the Reynolds number. The ratio $u_{\tau_{r}} / u_{\tau_{s}}$, see inset of figure 8 , increases with $R e_{\tau_{r}}$, consistently with the reduction of the skin-friction coefficient at the smooth wall, as $R e_{\tau_{s}}$ increases. HL and Ikeda (2002) (who had $R e_{\tau_{r}} \simeq 5600$ and 900, respectively) reported values of $u_{\tau_{r}} / u_{\tau_{s}}$ as large as $\simeq 2$, for $\lambda / k=10$.

The locations $y_{u v}$, where $\overline{u v}$ crosses zero, are almost indistinguishable from $y_{U}$ (figure 8), and therefore no region of negative turbulent energy production is expected. This point warrants further analysis since, as noted in the introduction, the evidence gathered so far in the asymmetric channel is somewhat inconclusive. It was pointed out earlier that the location of $y_{u v} \simeq y_{\tau}$ is controlled by the ratio of the shear stress coefficients at the two walls. The actual expression is obtained from the ratio of (2.7) and (2.8), namely

$$
\frac{y_{\tau}}{2 H}=\frac{\tau_{r}}{\tau_{r}-\tau_{s}}=\frac{u_{\tau_{r}}^{2}}{u_{\tau_{r}}^{2}+u_{\tau_{s}}^{2}} .
$$

While, arguably, $u_{\tau_{r}}^{2}$ becomes constant (when $R e_{\tau_{r}}$ exceeds a critical, low value), the behaviour of $u_{\tau_{s}}^{2}$ is more complex (e.g. figure 7.10 of Pope 2000). This latter quantity first decreases in the laminar regime, then increases during transition before finally decreasing in the fully turbulent regime. So $y_{\tau}$ must follow a similar but opposite trend, see (4.1), shifting back and forth between the two walls. Moreover, it is plausible that, as $R e_{\tau_{r}}$ increases, the variation of $y_{U}$ lags behind that of $y_{u v}$, since the former is related to a first-order quantity (i.e. the mean velocity). For example, $y_{U}$ could increase monotonically from $H / 2$ - the solution for symmetric walls - to the asymptotic value for large $R e_{\tau_{r}}$. If this is the case, there may be ranges of $R e_{\tau_{r}}$ values for which the two locations do not match and $y_{u v}>y_{U}$, yielding regions of negative energy production. In addition, one should not neglect the effect of $\lambda / k$ or that of the roughness shape on the difference between $y_{u v}$ and $y_{U}$, as shown in Leonardi et al. (2005). In this respect, it would be interesting to assess systematically the behaviour of $y_{u v}$ and $y_{U}$ in an asymmetric channel with two different rough walls.

Fluctuation intensities - normalized by the scales $u_{\tau_{r}}$ and $y_{u v}$ - are represented in figure 9. The effect of the Reynolds number is still appreciable, particularly on the streamwise component. This is surprising since, as noted earlier, $\left(u_{\tau_{r}} / U_{M}\right)^{2}$ is constant, in the present range of $R e_{\tau_{r}}$. One can conclude that, although the drag coefficient at the rough wall is largely independent of the Reynolds number, the profiles of the turbulence intensities (particularly for $u$ ) close to the wall are not. Applying the same normalization, HL obtained a comparable degree of universality for their profiles (also included in figure 9). Note that although they had $\lambda / k=10$, their data are not dissimilar from ours.

It is useful to compare our turbulent profiles with those in a smooth-wall channel. The data of Moser, Kim \& Mansour (1999) for a smooth-wall channel at $R e_{\tau}=590$ 


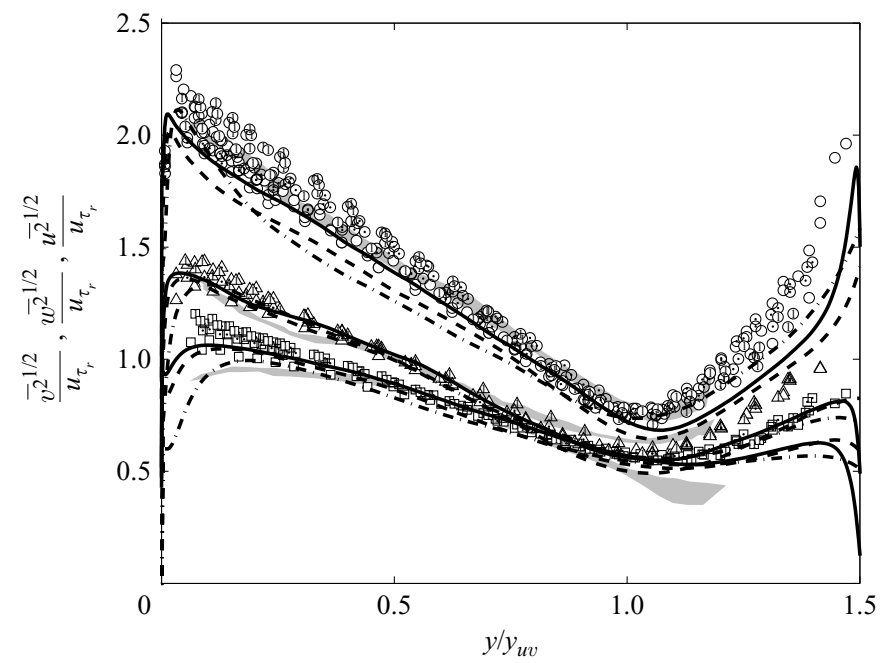

FIGURE 9. RMS turbulence intensities normalized by $u_{\tau_{r}}$ and $y_{u v}$. Symbols: experiment (data from single and X-wire). Lines: simulations; shaded regions: data from $\mathrm{HL}$ for $R_{b}=18500-56000$ and $\lambda / k=10$. Empty symbols: E1; dotted symbols: E2; cut symbols: $\mathrm{E} 3 ; \mathrm{O}, u ; \square, v ; \triangle, w ; \cdot-, \mathrm{D} 1 ;--, \mathrm{D} 2 ;-, \mathrm{D} 3$.

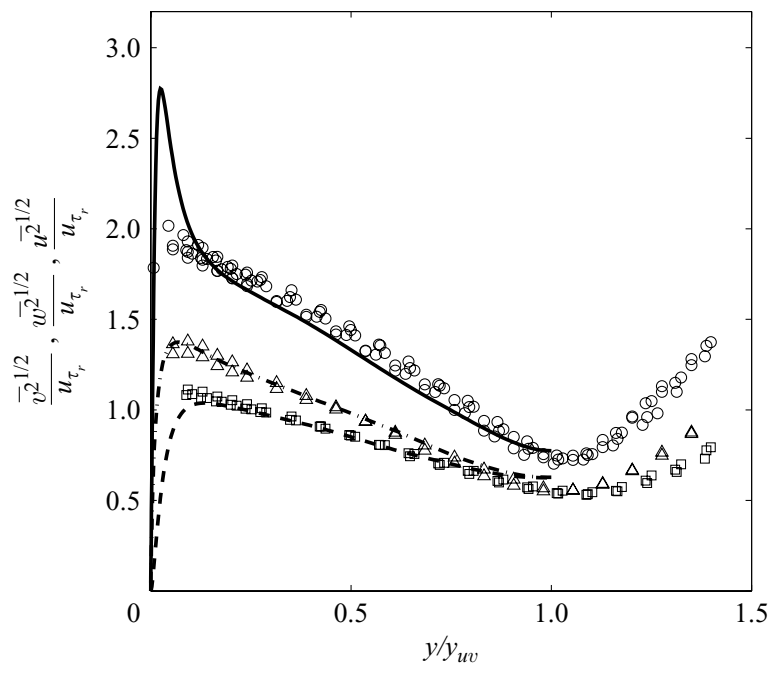

FIGURE 10. RMS turbulence intensities compared to the smooth-wall data of Moser et al.'s (1999) simulations at $R e_{\tau}=590$. Symbols: present data E2, normalized by $u_{\tau_{r}}$ and $y_{u v} ; \bigcirc, u$; $\square, v ; \triangle, w$. Lines: from Moser et al. (1999), normalized by $u_{\tau}$ and $H ; \cdot-, w ;--, v ;-, u$.

are plotted along with case E2 $\left(R e_{\tau_{r}}=620\right)$, in figure 10 . Near the roughness, the peak of $\overline{u^{2}}$ is largely reduced, most likely because of the shortening of the largest structures, as also pointed out by Bakken et al. (2005). However, when the normalized value of $y$ exceeds $\simeq 0.05$, the agreement between smooth- and rough-wall channel data is good for all the normal Reynolds stresses. Values at higher $\operatorname{Re}_{\tau}(=950)$ from del Alamo et al. (2004), figure 11, highlight the same type of agreement, perhaps extending even closer to the rough-wall. Hoyas \& Jiménez (2005) reported that, in the smooth channel, the profiles of $\overline{u^{2}}$ are still evolving in the range $R e_{\tau}=500-2000$ 


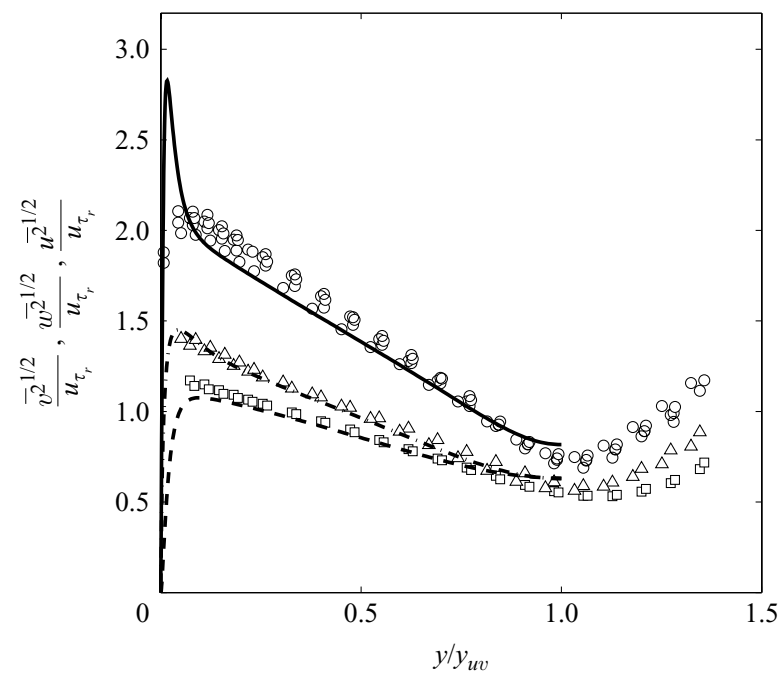

FIGURE 11. RMS turbulence intensities compared to the smooth-wall data of del Alamo et al.'s (2004) simulations at $R e_{\tau}=950$. Symbols: present data E2, normalized by $u_{\tau_{r}}$ and $y_{u v} ; \bigcirc, u$; $\square, v ; \triangle, w$. Lines: from del Alamo et al. (2004), normalized by $u_{\tau}$ and $H ; \cdot-, w ;--, v ;-, u$.

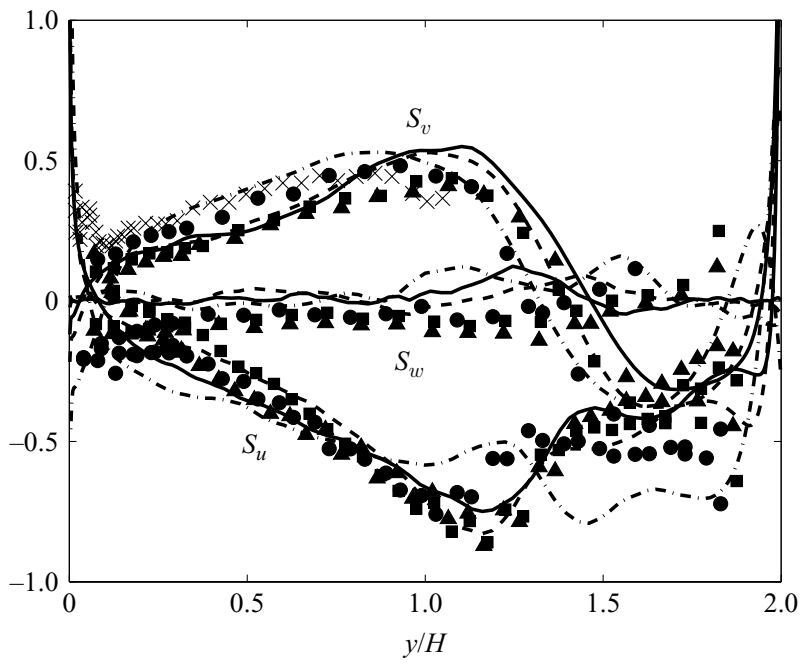

FIGURE 12. Skewness of $u, v$ and $w . \bullet, \mathrm{E} 1 ; \mathbf{\square}, \mathrm{E} 2 ; \mathbf{\Delta}, \mathrm{E} 3 ;--, \mathrm{D} 1 ;--, \mathrm{D} 2 ;-, \mathrm{D} 3$; $\times, S_{v}$ from Nakagawa et al. (2003) over a wavy wall.

and for all values of $y$, when normalized by $u_{\tau}^{2}$ and $H$. By comparison, $\overline{v^{2}}$ and $\overline{w^{2}}$ were found to be less dependent on $R e_{\tau}$. From figures 9-11, it could therefore be argued that the turbulent profiles are much less sensitive to the Reynolds number in the rough-wall case, possibly making modelling of this flow easier to achieve.

The skewness, $S_{u}=\bar{u}^{3} / \bar{u}^{3 / 2}$, and similarly for the $v$ and $w$ components, is plotted in figure 12. Since the single-wire $u$ data are in close agreement with the X-wire data, only these latter are plotted, to avoid crowding. For each component, the distributions are rather similar at different Reynolds numbers, in particular close to rough wall. $S_{u}$ is negative over almost the entire $y$ range, except very close to the walls, as indicated 


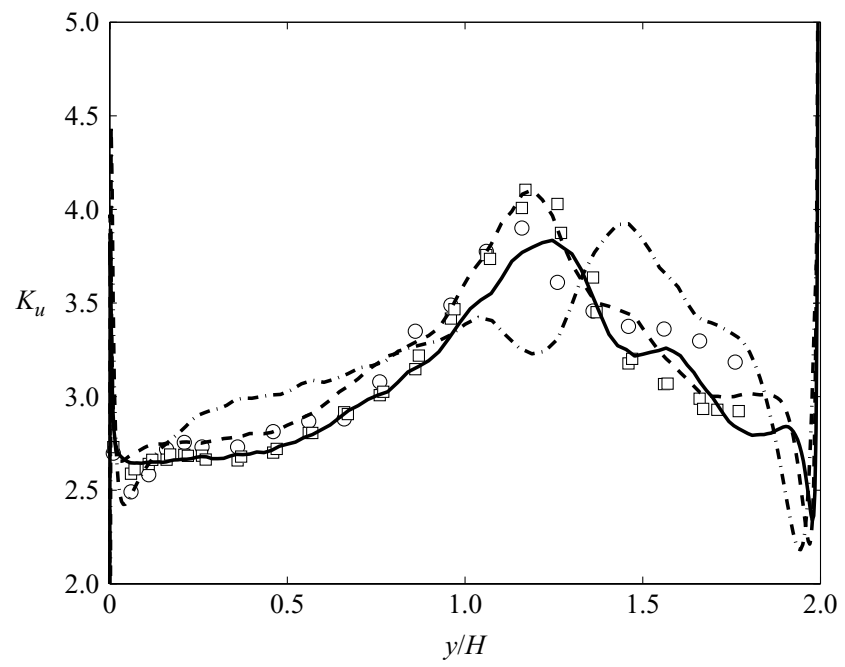

FIGURE 13. Kurtosis of $u . \mathrm{O}, \mathrm{E} 1 ; \square, \mathrm{E} 2 ; \triangle, \mathrm{E} 3 . \cdot-, \mathrm{D} 1 ;--, \mathrm{D} 2 ;-, \mathrm{D} 3$.

by DNS (very near the walls, the experimental data are not available) and reaches a minimum near $y_{u v}$. The profile of $S_{v}$ is roughly antisymmetric around $y_{u v}$, taking positive values near the rough wall. $S_{w}$ is practically zero everywhere, as it should be for symmetry. Although our profiles of $S_{v}$ match those reported by Nakagawa et al. (2003) in an asymmetric channel with one wavy wall (see figure 12), they differ substantially from those in HL, where $S_{v}$ is nearly coincident with $S_{u}$. The present profiles of the three skewnesses are however fully consistent with those in the symmetrically roughened channel of Ashrafian \& Andersson (2006b). The kurtosis of $u, K_{u}=\overline{u^{4}} /{\overline{u^{2}}}^{2}$ (figure 13), has values close to 3 (that of the normal distribution) for a wide region near the rough wall, also noted by HL.

Figure 14 shows the Taylor microscale $\lambda_{u}=\left(\overline{u^{2}} / \overline{(\partial u / \partial x)^{2}}\right)^{1 / 2}$. The numerical and experimental distributions have similar trends, with a maximum near $y=H$. The Kolmogorov length scale, plotted in figure 15, exhibits a maximum close to $y_{U}$, while decreasing almost linearly on the two sides of the peak. (Incidentally, this suggests a strategy for refining the numerical grid in the wall-normal direction, to achieve optimal resolution of the smallest scales.) Note that the larger difference between E1 and D1 is due to the disparity in the Reynolds number between these two cases. The resolution of the single wire can be estimated from the vertical scale on the right of figure 15, where $\eta$ is normalized with the length of the wire. $R_{\lambda}=\lambda_{u}{\overline{u^{2}}}^{1 / 2} / \nu$ (figure 16) attains larger values on the rough-wall side, particularly for E2 and E3; for this latter case, the maximum is near 230, at $y / H \simeq 0.5$. The comparison with the smooth-wall channel flow of Moser et al. (1999) and del Alamo et al. (2004), respectively at $R_{\tau}=590$ and 950, reinforces this conclusion (see also Abe, Kawamura \& Choi 2004a,b).

\subsection{Energy budget}

The turbulent kinetic energy budget in the channel is

$$
\frac{1}{2} \frac{\mathrm{d} \overline{q^{2}}}{\mathrm{~d} t}=0=\mathscr{P}+\mathscr{T}+\Psi+\mathscr{D}-\epsilon,
$$




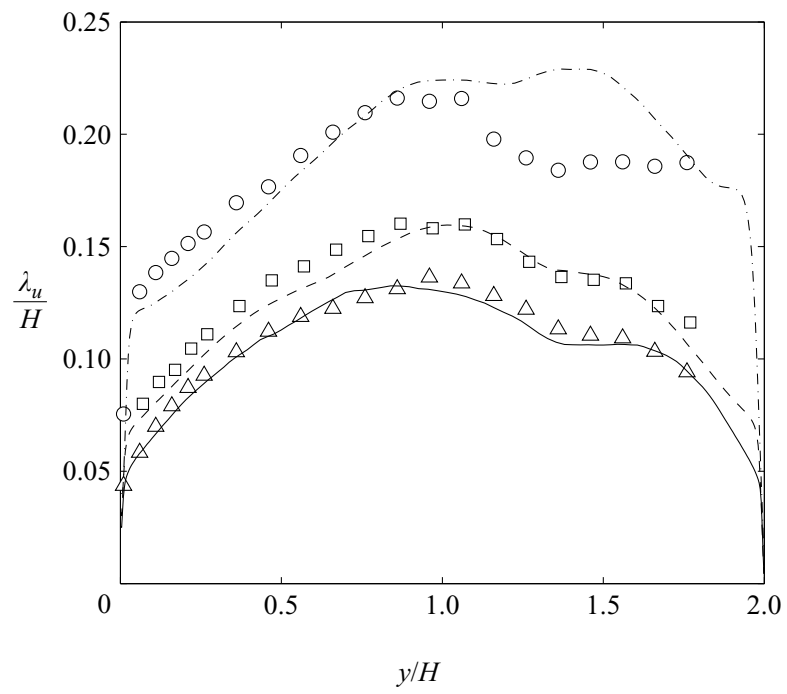

FIGURE 14. Taylor microscale. Symbols: experiments; lines: simulations. $\mathrm{O}, \cdot-, \mathrm{E} 1, \mathrm{D} 1 ;--, \square, \mathrm{E} 2, \mathrm{D} 2 ; \triangle,-, \mathrm{E} 3, \mathrm{D} 3$.

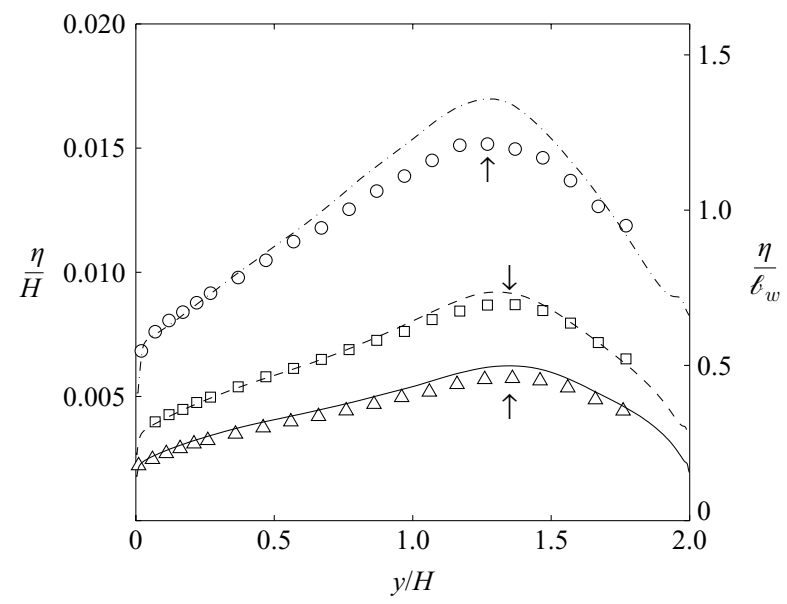

FigURE 15. Kolmogorov length scale. Symbols: experiments; lines: simulations. $\bigcirc,--$, E1, D1; ,$-- \square$, E2, D2; $\triangle,-$, E3, D3. The right-hand scale refers to the experimental values, as measured with the single wire of length $\ell_{w}=1.26 \mu \mathrm{m}$, used for the normalisation. Arrows indicate $y_{U} / H$ from experiments.

where $\overline{q^{2}}=\overline{u^{2}}+\overline{v^{2}}+\overline{w^{2}}$ is the total turbulent energy and

$$
\begin{aligned}
\mathscr{P} & =-\overline{u v} \frac{\partial U}{\partial y}, \\
\mathscr{T} & =-\frac{1}{2} \frac{\partial \overline{q^{2} v}}{\partial y}, \\
\Psi & =-\frac{\partial \overline{p v}}{\partial y},
\end{aligned}
$$




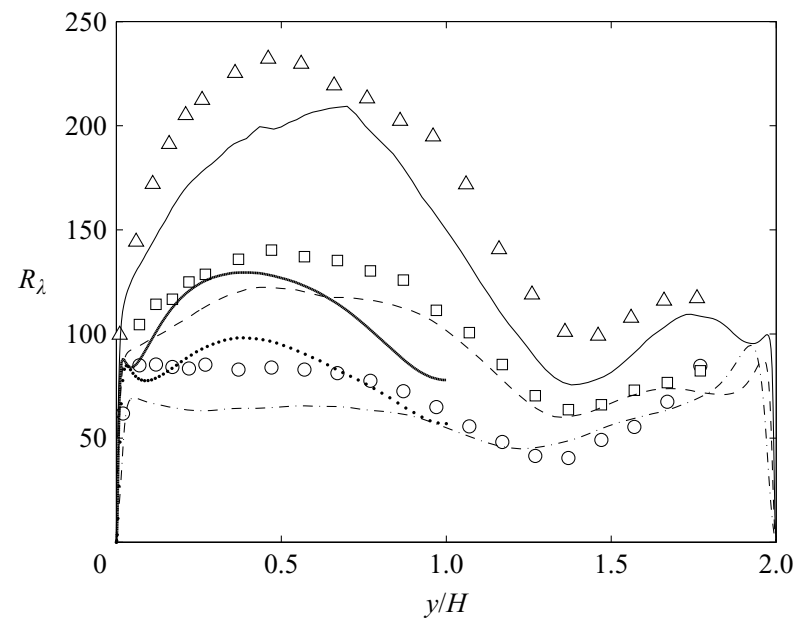

FIGURE 16. Taylor-microscale Reynolds number. Symbols: experiments; lines: simulations. $\mathrm{O}, \cdot-, \mathrm{E} 1, \mathrm{D} 1 ;--, \square, \mathrm{E} 2, \mathrm{D} 2 ; \triangle,-, \mathrm{E} 3$, D3. Thick dotted line, data from the smooth-wall channel flow of Moser et al. (1999) at $R_{\tau}=590$; thick solid line, data from the smooth-wall channel flow of del Alamo et al. (2004) at $R_{\tau}=950$.

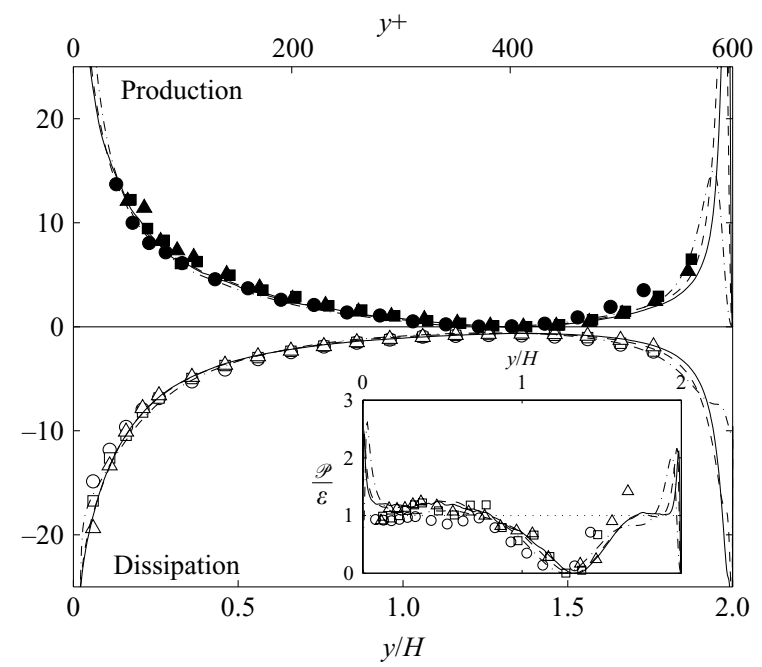

FIGURE 17. Major terms in the turbulent kinetic energy budget. Mean energy dissipation rate $-\epsilon H / u_{\tau_{r}}^{3}$ (empty symbols and thin lines); energy production $\mathscr{P} H / u_{\tau_{r}}^{3}$ (filled symbols and thick lines). $\bullet_{r}, \mathrm{O}, \mathrm{E} 1 ; \boldsymbol{\square}, \square, \mathrm{E} 2 ; \boldsymbol{\Delta}, \triangle, \mathrm{E} 3 ; \cdot-, \mathrm{D} 1 ;--, \mathrm{D} 2 ;-$, D3. Top abscissa scale refers to case E1. Inset: ratio of production to dissipation terms (key as in main figure).

$$
\mathscr{D}=-\frac{1}{2} \nu \frac{\partial^{2} \overline{q^{2}}}{\partial y^{2}}
$$

are the turbulent production, turbulent diffusion, pressure diffusion and viscous diffusion. In (4.2), the effect of the dispersive stress has been neglected. Although the pressure term is not measurable, the DNS of Leonardi et al. (2006a) shows that its effect is important only very close to the wall. Among the other terms, the major contributors are production and dissipation (figure 17). The balance between $\mathscr{P}$ and 


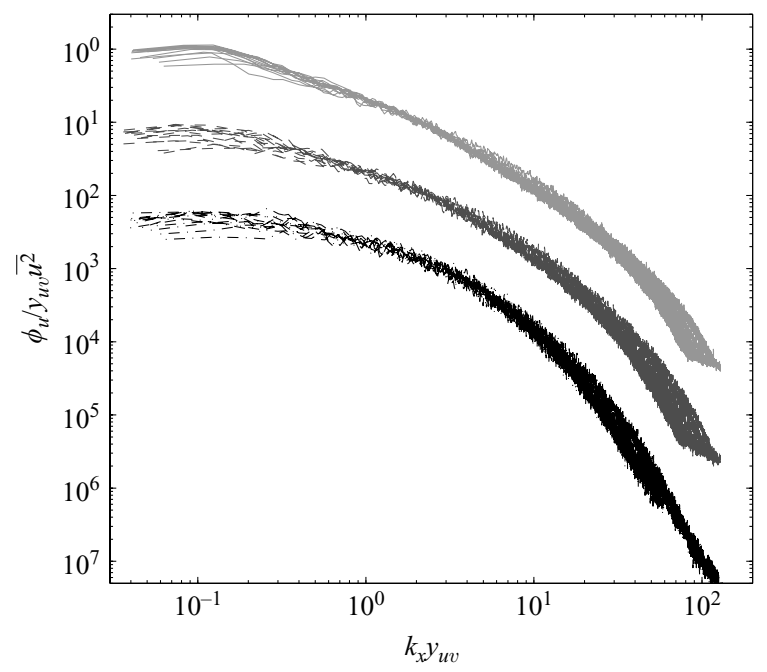

FIGURE 18. Spectra of $u$ in the range $0.14 \lesssim y / y_{u v} \lesssim 1$ and normalized by $\overline{u^{2}}$ and $y_{u v}$. - - E1 (shifted downwards by 2 decades); grey --, E2 (shifted downwards by 1 decade); grey - E3.

$\epsilon$ is almost complete (their ratio is close to 1 , as shown in the inset of figure 17) in the region $0.1 \lesssim y / H \lesssim 0.9$. It can be noted that the centre of this range corresponds to where $R_{\lambda}$ is maximum (figure 16). In a boundary layer, $\mathscr{P} \simeq \epsilon$ was associated by Townsend (1961) with the presence of an equilibrium layer. For E1, this region corresponds to $50 \lesssim y^{+} \lesssim 280$ (see the horizontal axis at the top of figure 17). Energy budgets from numerical studies of a channel flow with wall roughness (Nagano et al. 2004; Miyake et al. 2001; Ikeda 2002) confirm the dominant role of production and dissipation, in the above-mentioned range. Another conclusion to be drawn from the comparison with the DNS data is that the flow is, to a close approximation, locally isotropic (recall that $\epsilon_{i s o}$ is used instead of $\epsilon$ in the experiments).

\subsection{Spectra}

Distributions of $\phi_{u}\left(k_{x}\right)$, defined such that

$$
\overline{u^{2}}=\int_{0}^{\infty} \phi_{u}\left(k_{x}\right) \mathrm{d} k_{x}
$$

are shown in figure 18. These data, normalized by $\overline{u^{2}}$ and $y_{u v}$, have been measured with the single wire in the region $0.14 \lesssim y / y_{u v} \lesssim 1$. At each Reynolds number, the profiles collapse approximately onto a single curve, over the entire range of scales. Nakagawa et al.'s (2003) spectra, measured over a wavy wall, display the same level of collapse, for approximately the same locations. As $R e_{\tau_{r}}$ is increased, the shape of the spectrum changes and a scaling range becomes more evident. This is clearer in figure 19, where the normalization is on Kolmogorov scales. The collapse of the spectra is rather good at large wavenumbers (in the dissipative range) at all $R e_{\tau_{r}}$, and extends to smaller wavenumbers with increasing $R e_{\tau_{r}}$. The spectra for a location near $y \simeq 0.5 H$ (where $R_{\lambda}$ is largest) are plotted in the inset of figure 19, after multiplication by $\left(k_{x} \eta\right)^{5 / 3}$, in order to highlight the inertial range. Although, as $R_{\lambda}$ increases, the maximum tends to flatten, a wide plateau has not yet formed. This is expected, however, considering the low values of $R_{\lambda}$. 


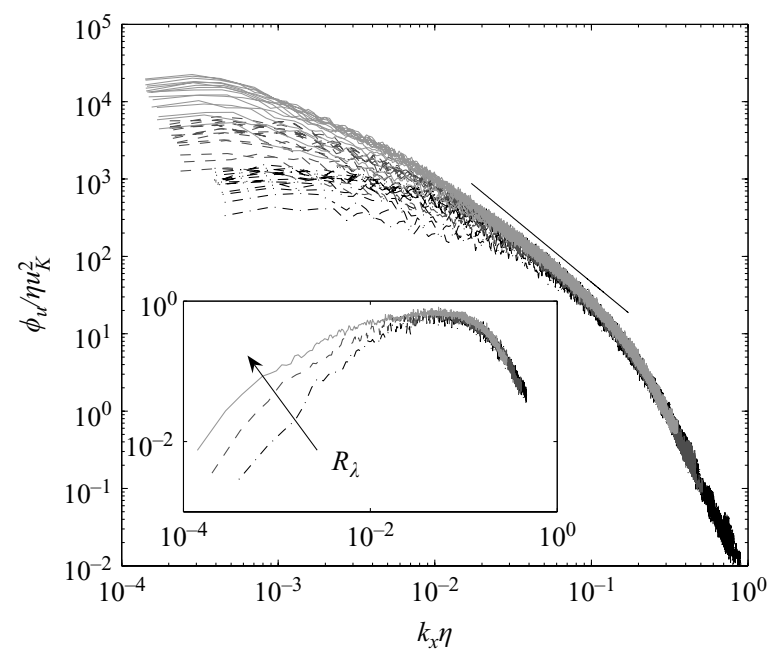

FIGURE 19. Spectra of $u$ in the range $0.1 \gtrsim y / H \gtrsim 1$ and normalized by Kolmogorov scales $u_{K}^{2}$ and $\eta . \cdot-$, E1; grey -- , E2; grey - , E3. The straight line has a slope of $-5 / 3$. The inset shows the spectra at $y \simeq 0.5 H$ multiplied by $\left(k_{x} \eta\right)^{5 / 3}$.

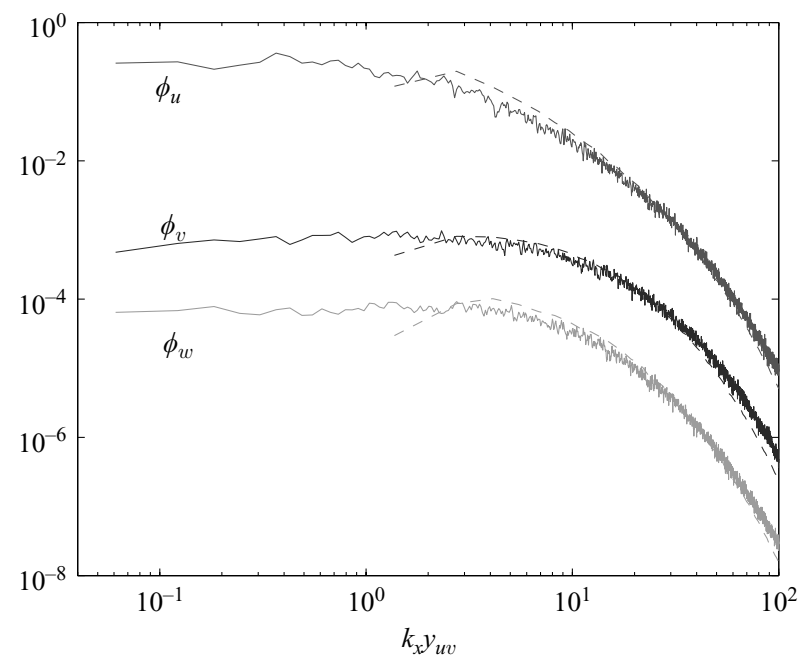

FIGURE 20. Spectra of $u, v$ and $w$ at $y / H=0.2$ for E1 (solid lines) and D1 (dashed lines), normalized by the local turbulence intensity and $y_{u v}$. (The distributions of $v$ and $w$ are shifted down by 2 and 3 decades.)

Numerical and experimental spectra are compared in figures 20-22. All three velocity components are considered at one location $(y / H=0.2)$ in the equilibrium layer. Note that the DNS spectra are computed from the spatial distributions of the velocity in the streamwise direction and, therefore, do not require Taylor's hypothesis. For each component, reasonable agreement is observed between numerical and experimental data. Profiles at $R e_{\tau_{r}} \simeq 3600$ from HL (their figures 15-17) are also included in figure 22. While their spectra for $u$ and $v$ display very similar trends to the present $u$ and $v$ spectra, their $w$ spectrum differs from ours. Spectra of $u$ and $v$ measured by Nakagawa \& Hanratty (2003) over a wavy-wall channel flow at 


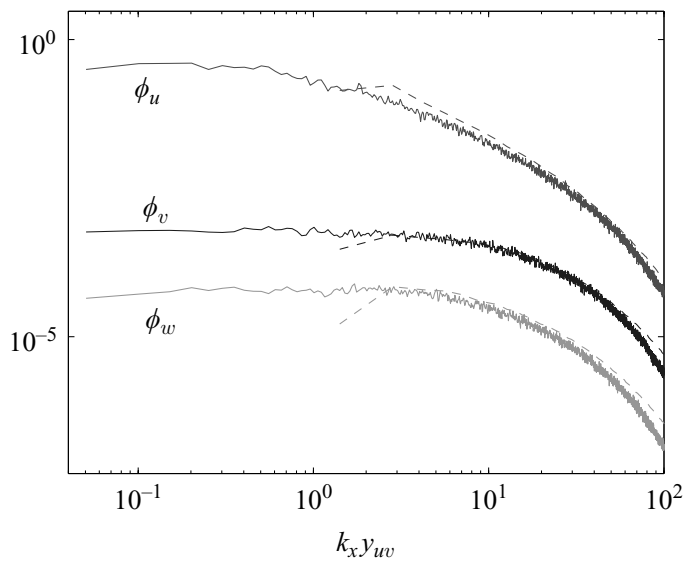

Figure 21. Spectra of $u, v$ and $w$ at $y / H=0.2$ for E2 (solid lines) and D2 (dashed lines), normalized by the local turbulence intensity and $y_{u v}$. (The distributions of $v$ and $w$ are shifted down by 2 and 3 decades.)

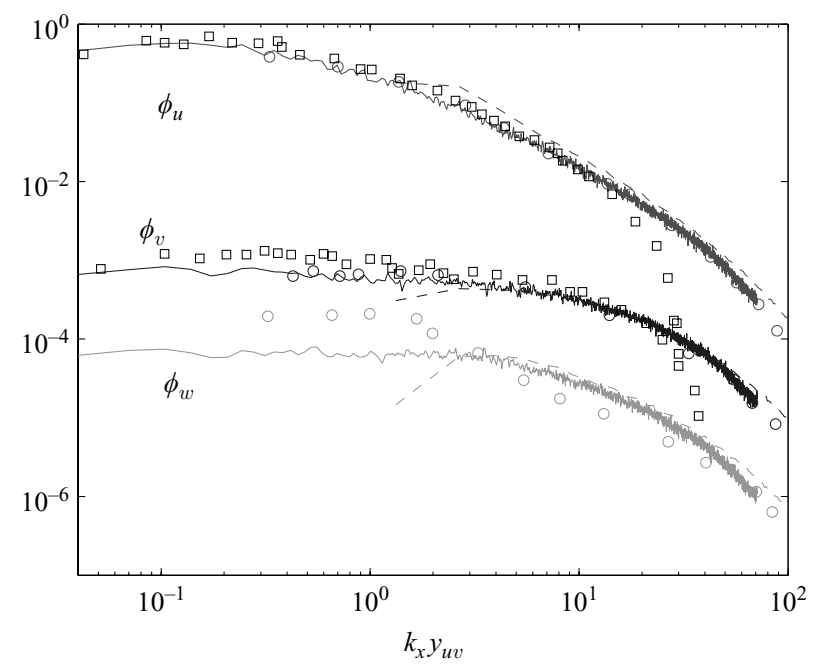

Figure 22. Spectra of $u, v$ and $w$ at $y / H=0.2$ for E3 (solid lines) and D3 (dashed lines), normalized by the local turbulence intensity and $y_{u v}$. (The distributions of $v$ and $w$ are shifted down by 2 and 3 decades.) $\bigcirc$ : data from $H L$ at $y / H \simeq 0.2$ for $R e_{\tau_{r}} \simeq 3650$; $\square$ : data from Nakagawa \& Hanratty (2003) at $y / H \simeq 0.24$ for $\operatorname{Re}_{b}=11000$.

$R e_{b}=11000$ are also included in the figure. Agreement with our spectra is good only up to $k_{x} y_{u v} \simeq 20$, while the intensity of the fluctuations at larger wavenumbers is much lower over the wavy wall (since these spectra were measured with a laser Doppler anemometer, some underestimation of the velocity fluctuations at large $k$ should be expected.) Despite this, a conclusion that can be drawn from the comparison is that a consistent portion of turbulent scales, physically located in the equilibrium layer, has a similar energy distribution for different boundary conditions, when scaled with the variables $\overline{u^{2}}$ and $y_{u v}$. This could prove useful in modelling the large scales in this flow type. 


\section{Conclusions}

Several aspects of the flow in an asymmetric channel, with roughness elements on one side, have been described in the present work. Experiments and direct numerical simulations, carried out for the same geometrical and flow conditions, have provided data for turbulence intensities, length scales, major terms in the turbulent energy equation, and spectra. The close agreement between experimental and numerical results serves to reinforce the present conclusions. When the normalization is based on outer variables - the rough-wall shear velocity and the zero-crossing location $y_{u v}$ of the Reynolds shear stress - the magnitude of the turbulence intensities on the rough-wall side of the channel evolves marginally as $R e_{\tau_{r}}$ increases between 300 and 1100. This is consistent with the independence of $R e_{\tau_{r}}$ of the rough-wall friction coefficient. The normalization on outer variables is also able to bring the present distributions into accord with those for a smooth-wall channel at similar $R e_{\tau}$, except for a small region near the wall. Although the results in this paper pertain to a particular roughness pitch-to-height ratio, our conclusion regarding the Reynolds number independence should apply equally to other values of this ratio, provided the pressure distribution around the elements remains the major contributor to the drag.

The turbulent energy budget shows that the turbulent energy production is always positive. Further, the energy dissipation rate and production terms are found to be dominant and approximately in balance in a wide region $\left(0.1 \lesssim y / y_{u v} \lesssim 0.7\right)$ on the rough-wall side of the channel. The isotropy of the small scales, assumed when estimating the measured mean energy dissipation rate near the rough wall, appears to be a valid working hypothesis. This is also commensurate with the higher values of the Taylor-microscale Reynolds number achieved on the rough-wall side of the channel. Across almost the entire wavenumber range and for $0.14 \lesssim y / y_{u v} \lesssim 1$, the spectra of $u$ collapse approximately when normalized on the local longitudinal turbulence intensity and $y_{u v}$, for any fixed value of $R e_{\tau_{r}}$. As the Reynolds number varies, this normalization becomes ineffective, and there is little doubt that Kolmogorov normalization provides a better description of the dissipative range and at least the beginning of the inertial range.

S. L. is grateful to the National Science Foundation for providing computation time through the TeraGrid resources SDSC and TACC (grant n. CTS070005T). P. O. acknowledges the support of a MIUR $60 \%$ grant, a PRIN to purchase a cluster of PCs, and CASPUR for providing computing resources.

\section{REFERENCES}

Abe, H., Kawamura, H. \& Chol, H. 2004a Surface heat-flux fluctuations in a turbulent channel flow up to up to $R e_{\tau}=1020$ with $P r=0.025$ and 0.71. Intl $J$. Heat Fluid Flow 25, 404-419.

Abe, H., Kawamura, H. \& ChOI, H. 2004b Very large-scale structures and their effects on the wall shear-stress fluctuations in a turbulent channel flow up to $R e_{\tau}=640$. Trans. ASME: J. Fluids Engng 126, 835-843.

del Alamo, J. C., Jimenez, J., Zandonade, P. \& Moser, R. D. 2004 Scaling of the energy spectra of turbulent channels. J. Fluid Mech. 500, 135-144.

Ashrafian, A. \& Andersson, H. I. $2006 a$ Roughness effects in turbulent channel flow. Prog. Comput Fluid Dyn. 6, 1-20.

Ashrafian, A. \& ANDERsson, H. I. 2006 $b$ The structure of turbulence in a rod-roughened channel. Intl J. Heat Fluid Flow 27, 65-79.

Ashrafian, A., Andersson, H. I. \& Manhart, M. 2004 DNS of turbulent flow in a rod-roughened channel. Intl J. Heat Fluid Flow 25, 373-383. 
Bakken, O. M., Krogstad, P.-Å., Ashrafian, A. \& Andersson, H. I. 2005 Reynolds number effects in the outer layer of the turbulent flow in a channel with rough walls. Phys. Fluids 17, 065101.

Bhaganagar, K., Kim, J. \& Coleman, G. 2004 Effect of roughness on wall-bounded turbulence. Flow Turbul. Combust. 72, 463-492.

Burattini, P. \& Antonia, R. A. 2005 The effect of different X-wire calibration schemes on some turbulence statistics. Exps. Fluids 38, 80-89.

Castillo, L., Seo, J., Hangan, H. \& Johansson, T. G. 2004 Smooth and rough turbulent boundary layers at high Reynolds number. Exps. Fluids 36, 759-774.

Chang, S. W., Liou, T.-M. \& Juan, W.-C. 2005 Influence of channel height on heat transfer augmentation in rectangular channels with two opposite rib-roughened walls. Intl J. Heat Mass Transfer 48, 2806-2813.

Cheng, H. \& Castro, I. P. 2002 Near wall flow over urban-like roughness. Boundary-Layer Met. 104, 229-259.

Cherukat, P., Na, Y., Hanratty, T. J. \& Mclaughlin, J. B. 1998 Direct numerical simulation of a fully developed turbulent flow over a wavy wall. Theor. Comput. Fluid Dyn. 11, 109-134.

Chung, S. Y., Rhee, G. H. \& Sung, H. J. 2002 Direct numerical simulation of turbulent concentric annular pipe flow. Intl J. Heat Fluid Flow 23, 426-440.

Cui, J., Patel, V. C. \& Lin, C.-L. 2003 Large-eddy simulation of turbulent flow in a channel with rib roughness. Intl J. Heat Fluid Flow 24, 372-388.

Djenidi, L., Elavarasan, R. \& Antonia, R. A. 1999 The turbulent boundary layer over transverse square cavities. J. Fluid Mech. 395, 271-294.

Finnigan, J. 2000 Turbulence in plant canopies. Annu. Rev. Fluid Mech. 32, 519-571.

Furuya, Y., Miyata, M. \& Fujita, H. 1976 Turbulent boundary layer and flow resistance on plates roughened by wires. Trans. ASME: J. Fluids Engng 98, 635-644.

Hanjalić, K. \& Launder, B. E. 1972 Fully developed asymmetric flow in a plane channel. J. Fluid Mech. 51, 301-335.

HoyAs, S. \& JimÉneZ, J. 2005 Scaling of the velocity fluctuations in turbulent channels up to $R e_{\tau}=2003$. CTR Annual Research Briefs.

IKeDA, T. 2002 Direct simulation of a rough-wall channel flow. PhD thesis, Stanford University.

Ikeda, T. \& Durbin, P. A. 2007 Direct simulations of a rough-wall channel flow. J. Fluid Mech. 571, 235-263.

Jakirlí́, S., Hanjalíc, K. \& Tropea, C. 2002 Modeling rotating and swirling turbulent flows: A perpetual challenge. AIAA J. 40, 1984-1996.

JimÉneZ, J. 2004 Turbulent flows over rough walls. Annu. Rev. Fluid Mech. 36, 173-196.

Krogstad, P.-Å., Andersson, H. I., Bakken, O. M. \& Ashrafian, A. 2005 An experimental and numerical study of channel flow with rough walls. J. Fluid Mech. 530, 327-352.

Krogstad, P.-Å. \& Antonia, R. A. 1994 Structure of turbulent boundary layers on smooth and rough walls. J. Fluid Mech. 277, 1-21.

LEE, S.-H. \& SUNG, H. J. 2007 Direct numerical simulation of the turbulent boundary layer over a rod-roughened wall. J. Fluid Mech. 584, 125-146.

LeONARDi, S., Orlandi, P. \& Antonia, R. A. 2005 A method for determining the frictional velocity in a turbulent channel flow with roughness on the bottom wall. Exps. Fluids 38, 796-800.

Leonardi, S., Orlandi, P., Djenidi, L. \& Antonia, R. A. 2004 Structure of turbulent channel flow with square bars on one wall. Intl J. Heat Fluid Flow 25, 384-392.

Leonardi, S., Orlandi, P., Duenidi, L. \& Antonia, R. A. $2006 a$ Guidelines for modeling a 2D rough wall channel flow. Flow Turb. Combust. 77, 41-57.

Leonardi, S., Orlandi, P., Smalley, R. J., Djenidi, L. \& Antonia, R. A. 2003 Direct numerical simulations of turbulent channel flow with transverse square bars on one wall. J. Fluid Mech. 491, 229-238.

Leonardi, S., Tessicini, F., Orlandi, P. \& Antonia, R. A. $2006 b$ Direct numerical and large-eddy simulations of turbulent flows over rough surfaces. AIAA J. 44, 2482-2487.

Liberzon, A., Lthi, B., Guala, M., Kinzelbach, W. \& Tsinober, A. 2005 Experimental study of the structure of flow regions with negative turbulent kinetic energy production in confined three-dimensional shear flows with and without buoyancy. Phys. Fluids 17, 095110.

Martinez, D. O., Chen, S., Doolen, G. D., Kraichnan, R. H., Wang, L.-P. \& Zhou, Y. 1997 Energy spectrum in the dissipation range of fluid turbulence. J. Plasma Phys. 57, 195-201. 
Mathieu, J. \& Scott, J. 2000 An Introduction to Turbulent Flow. Cambridge University Press.

Miyake, Y., Tsujimoto, K. \& Nagai, N. 2002 Numerical simulation of channel flow with a ribroughened wall. J. Turb. 3, 035.

Miyake, Y., Tsujimoto, K. \& NaKaji, M. 2001 Direct numerical simulation of rough-wall heat transfer in a turbulent channel flow. Intl J. Heat Fluid Flow 22, 237-244.

Moser, R. D., Kim, J. \& Mansour, N. N. 1999 DNS of turbulent channel flow up to $R e_{\tau}=590$. Phys. Fluids 11, 943-945.

Nagano, Y., Hattori, H. \& Houra, T. 2004 DNS of velocity and thermal fields in turbulent channel flow with transverse-rib roughness. Intl J. Heat Fluid Flow 25, 393-403.

NakaGaWA, S. \& HaNRATTY, T. J. 2003 Influence of a wavy boundary on turbulence. II. Intermediate roughened and hydraulically smooth surfaces. Exps. Fluids 35, 437-447.

Nakagawa, S., Na, Y. \& Hanratty, T. J. 2003 Influence of a wavy boundary on turbulence. I. Highly rough surface. Exps. Fluids 35, 422-436.

Orlandi, P. 1999 Fluid Flow Phenomena. A Numerical Toolkit. Kluwer.

ORLANDI, P. \& LEONARDI, S. 2006 DNS of turbulent channel flows with two- and three-dimensional roughness. J. Turb. 7, 1-22.

Perry, A. E., Schofield, W. H. \& Joubert, P. N. 1969 Rough wall turbulent boundary layers. $J$. Fluid Mech. 37, 383-413.

Pope, S. B. 2000 Turbulent Flows. Cambridge University Press.

Raupach, M. R., Antonia, R. A. \& Rajagopalan, S. 1991 Rough-wall turbulent boundary layers. Appl. Mech. Rev. 44, 1-25.

SNYDER, W. H. \& CASTRO, I. P. 2002 The critical Reynolds number for rough-wall boundary layers. J. Wind Engng Ind. Aerodyn. 90, 41-54.

Thurlow, E. M. \& Klewicki, J. C. 2000 Experimental study of turbulent Poiseuille-Couette flow. Phys. Fluids 12, 865-875.

Townsend, A. A. 1961 Equilibrium layers and wall turbulence. J. Fluid Mech. 11, 97-120.

Vlachogiannis, M. \& Hanratty, T. J. 2004 Influence of wavy structured surfaces and large scale polymer structures on drag reduction. Exps. Fluids 36, 685-700.

WANG, L. \& Sunden, B. 2005 Experimental investigation of local heat transfer in a square duct with continuous and truncated ribs. Expl Heat Transfer 18, 179-197.

WyngaARD, J. C. 1968 Measurement of small-scale turbulence structure with hot wires. J. Phys. E: Sci. Instrum. 1, 1105-1108.

Zaman, K. B. M. Q. \& Hussain, A. K. M. F. 1980 Vortex pairing in a circular jet under controlled excitation. Part 1. General jet response. J. Fluid Mech. 101, 449-491. 